\title{
Auger cascades leading to higher charged states in xenon driven by an X-ray free-electron-laser pulse
}

\author{
Henry I. B. Banks, Antonis Hadjipittas, and Agapi Emmanouilidou ${ }^{\mathrm{a}}$ \\ Department of Physics and Astronomy, University College London, Gower Street, London WC1E 6BT, UK
}

Received 23 August 2019 / Received in final form 12 December 2019

Published online 21 May 2020

(C) The Author(s) 2020. This article is published with open access at Springerlink.com

\begin{abstract}
We study the interaction of xenon with an $850 \mathrm{eV}$ photon energy FEL pulse. We compute singlephoton ionisation cross sections and Auger rates by adopting to atoms a formalism we previously developed for diatomic molecules. In this formulation, a bound orbital is expressed as a sum of wave functions each corresponding to a different $l$ quantum number. In contrast, in previous formulations only one $l$ quantum number is associated with a bound orbital. As a result, in the non-relativistic regime, the description of the bound states is more accurate in our computations. Employing a Monte-Carlo technique, we find that our results for the ion state yields of xenon compare well with experimental results. Moreover, we find that when xenon is driven by two FEL pulses of the same energy but different pulse duration, higher-charged states are produced in the case of the longer duration and less intense laser pulse. An analysis of the ionization pathways reveals that less single-photon absorptions underlie the formation of each higher-charged state for the longer duration pulse compared to the shorter one. We find that the reason for the formation of higher-charged states for the longer duration pulse is the prevalence of Auger cascades.
\end{abstract}

\section{Introduction}

The development of X-ray free-electron lasers (XFELs) [1-3] constitutes a new tool for high-resolution probing of atoms and molecules $[4,5]$ and bio-molecular imaging [6-9]. The X-ray energy of these photons results in innershell electrons being more likely to ionise than valence ones. Inner-shell ionisation results in the creation of "hollow" states. Such states are of particular interest in molecules, due to the sensitivity of these states to their chemical environment $[10,11]$. A "hollow" state can decay quickly via an Auger process. That is, an electron from a higher energy sub-shell falls down to fill in a core hole and in doing so releases enough energy to ionise another electron. The new state, resulting from an Auger transition, may also be a "hollow" state itself, thus, triggering an Auger cascade. As we show, such Auger cascades can prevail in the interaction of xenon with FEL pulses.

FEL interactions with xenon have been the subject of many studies in recent years [12-17]. Namely, time-offlight experiments have measured the ion yields produced when xenon interacts with high-intensity FEL pulses at both low (extreme ultraviolet) [12] and high (X-ray) [13] photon energies. For low photon energies, using rate equations, insights into the resulting highly-charged ion states have been obtained by accounting, in addition to singlephoton, for multi-photon processes $[14,15]$. The cross sections for these multi-photon processes were obtained using

a e-mail: a.emmanouilidou@ucl.ac.uk scaling laws [18]. For X-ray photon energies, Monte-Carlo methods for multi-electron atoms have been employed to gain insight into the dynamics leading to the formation of highly-charged ion states $[13,17,19]$. Moreover, the interaction of xenon clusters with XFELs and the resulting ion yields have been addressed experimentally as well as theoretically [20].

In this work, we gain further insight into the formation of the final highly-charged ion states of xenon driven by an XFEL pulse by identifying the prevalent pathways for each ion state. A pathway refers to the sequence of states accessed in time due to the interaction of xenon with the pulse. Each state is described by its electronic configuration. To obtain the ion yields and the pathways underlying the formation of each ion state, we compute the single-photon ionisation cross sections and Auger decay rates for all energetically allowed transitions. We do so by adapting to atoms the formalism that we have previously developed for diatomic molecules [21]. Our formalism is general. It applies to complex multi-electron atoms with bound orbital wave functions that can be accurately described when accounting for several angular momentum $l$ quantum numbers. In contrast, the previous formalism we employed for computing single-photon ionisation cross sections and Auger rates for argon $[22,23]$ and the formalism employed in the XATOM-model [24] use bound orbital wave functions described by only one $l$ quantum number. Thus, the current formalism, allows for a more accurate computation of the single-photon ionization cross sections and Auger rates in the non-relativistic regime. 
We employed rate equations in our studies of argon interacting with FEL pulses in order to obtain the ion yields and prevalent pathways, [22,23]. Here, we employ a Monte-Carlo technique [25] to obtain ion yields and pathways for driven xenon. The reason is that 200 states are energetically accessed when argon interacts with a $315 \mathrm{eV}$ pulse while a significantly larger number of roughly $2 \times 10^{4}$ is accessed when xenon interacts with an $850 \mathrm{eV}$ pulse. Thus, $2 \times 10^{4}$ rate equations must be solved to obtain the ion yields of xenon. Moreover, the number of rate equations one must solve to identify the pathways of driven xenon is computationally prohibitive. We address this issue by employing a much faster Monte-Carlo technique where, starting from an initial state, only one state is accessed at a time, which is determined in a stochastic manner $[13,17,19]$.

Employing the single-photon ionisation cross sections and Auger rates in the Monte-Carlo technique, we find that our results for the ion yields of xenon interacting with an $850 \mathrm{eV}$ FEL pulse are in good agreement with experimental ones [20]. Moreover, we compute the prevalent pathways in the formation of the ion states of xenon. These pathways are more complex compared to the ones involved in argon when driven by a $315 \mathrm{eV}$ FEL pulse [22,23]. In the latter work, we found that even-charged ion states of argon were more populated than odd-charged ones. The reason is that once an inner-shell vacancy is created only one Auger decay follows. Thus, the prevalent pathways involve mostly sequences of a pair of an inner-shell singlephoton absorption and an Auger decay.

We find that in xenon driven by an $850 \mathrm{eV}$ FEL pulse the resulting pathways involve a much more complex sequence of single-photon ionization processes and Auger decays. We demonstrate this, by comparing the yields of the highly-charged ion states resulting from the interaction of xenon with two FEL pulses of the same pulse energy. For the longer duration pulse, we find larger yields for the highly-charged states. We show that this is due to Auger cascades taking place following the ionization of a $3 d$ electron by single photon absorption. That is, the $3 d$ hole is filled via an Auger process from an electron at an $n=4$ shell. Subsequently, more Auger processes can take place with electrons from $n=5$ shells dropping in to fill holes in $n=4$ shells. These cascades do not prevail in the short duration pulse. Instead, we find that, compared to the long duration pulse, more single-photon absorptions occur for a given highly-charged state.

\section{Method}

We employ a Monte-Carlo technique to obtain the yields of the final ion states and the pathways leading to the formation of each state. In our computations, neutral xenon and each ion state of xenon is defined by the electronic configuration $1 s^{a} 2 s^{b} 2 p^{c} 3 s^{d} 3 p^{e} 3 d^{f} 4 s^{g} 4 p^{h} 4 d^{k} 5 s^{l} 5 p^{m}$. Each of the $n p$ sub-shells involves orbitals $n p_{x}, n p_{y}, n p_{z}$, while each of the $n d$ sub-shells involves orbitals $n d_{x y}, n d_{y z}, n d_{x z}$, $n d_{x^{2}-y^{2}}, n d_{z^{2}}$. The maximum occupancy of each orbital is two. In what follows, we obtain the single-photon ionisation cross sections and Auger rates in terms of orbitals. We then sum over the relevant orbitals to obtain the respective rates between sub-shells. These latter rates are the ones employed in the Monte-Carlo technique.

\subsection{Single-photon ionisation}

We compute the single-photon ionisation cross section for an electron to transition from an initial bound orbital $\phi_{a}$ to a final continuum orbital $\phi_{\epsilon, l, m}$, with well-defined quantum number $l$ and magnetic quantum number $m$, as follows [26]:

$$
\sigma_{a \rightarrow \epsilon, l, m}=\frac{4}{3} \alpha \pi^{2} \omega N_{a} \sum_{M=-1,0,1}\left|D_{a \rightarrow \epsilon, l, m}^{M}\right|^{2} .
$$

We denote by $\alpha$ the fine structure constant, by $N_{a}$ the occupation number of the bound orbital $a$, by $\omega$ the photon energy, and by $M$ the polarisation of the photon. In the length gauge, the matrix element $D_{a \rightarrow \epsilon, l, m}^{M}$ is given by

$$
D_{a \rightarrow \epsilon, l, m}^{M}=\int \phi_{a}(\mathbf{r}) \phi_{\epsilon, l, m}^{*}(\mathbf{r}) \sqrt{\frac{4 \pi}{3}} r Y_{1 M}(\theta, \phi) d \mathbf{r} .
$$

For computational efficiency, we split the integral in equation (2) into a radial and an angular component, since the latter integral can be performed analytically. To do so, we express the orbital $\phi_{a}$ in terms of radial wave functions, $P_{a, l^{\prime}, m^{\prime}}(r)$, and spherical harmonics, $Y_{l^{\prime}, m^{\prime}}(\theta, \phi)$ as follows

$$
\phi_{a}(\mathbf{r})=\sum_{l^{\prime}, m^{\prime}} P_{a, l^{\prime}, m^{\prime}}(r) Y_{l^{\prime}, m^{\prime}}(\theta, \phi) / r .
$$

We compute the bound orbital wave functions using Molpro [27] with the AQZP [28] basis set, which results in an accurate description of the bound orbitals of xenon. Employing this basis set, each bound orbital is expressed as a combination of several $l^{\prime}, m^{\prime}$ terms. In contrast, in our studies for driven argon, each orbital had well defined $l, m$ numbers. The reason was that we employed the $6-311 \mathrm{G}$ basis set which was sufficient to adequately describe the bound orbitals of argon. Furthermore, we express the continuum orbital $\phi_{\epsilon, l, m}$ as follows

$$
\phi_{\epsilon, l, m}(\mathbf{r})=P_{\epsilon, l}(r) Y_{l, m}(\theta, \phi) / r .
$$

We compute the continuum wave functions, $\phi_{\epsilon, l, m}$, using the Numerov technique [29] with a Hartree-Fock-Slater (HFS) potential obtained from the Herman-Skillman code $[30,31]$. We also employed this technique to obtain continuum orbitals in previous studies of argon driven by an FEL pulse [22].

Substituting in equation (2) the expressions for $\phi_{a}(\mathbf{r})$ and $\phi_{\epsilon, l, m}(\mathbf{r})$ given by equations (3) and (4), respectively, we obtain

$$
\begin{aligned}
D_{a \rightarrow \epsilon, l, m}^{M}= & \sqrt{\frac{4 \pi}{3}} \sum_{l^{\prime}, m^{\prime}} \int_{0}^{\infty} d r P_{a, l^{\prime}, m^{\prime}}(r) r P_{\epsilon, l}^{*}(r) \\
& \times \int d \Omega Y_{l^{\prime}, m^{\prime}}(\theta, \phi) Y_{l, m}^{*}(\theta, \phi) Y_{1 M}(\theta, \phi) .
\end{aligned}
$$


Table 1. Single-photon ionisation cross sections for different sub-shells of xenon in the ground state interacting with an $850 \mathrm{eV}$ pulse.

\begin{tabular}{lll}
\hline Initial sub-shell & Ref. [33] $\left[\mathrm{cm}^{2}\right]$ & This Work $\left[\mathrm{cm}^{2}\right]$ \\
\hline $3 d^{10}$ & $2.05 \mathrm{e}-18$ & $1.61 \mathrm{e}-18$ \\
$4 s^{2}$ & $3.77 \mathrm{e}-20$ & $3.10 \mathrm{e}-20$ \\
$4 p^{6}$ & $1.27-19$ & $9.60 \mathrm{e}-20$ \\
$4 d^{10}$ & $2.34-19$ & $1.79 \mathrm{e}-19$ \\
$5 s^{2}$ & $6.04-21$ & $3.68 \mathrm{e}-21$ \\
$5 p^{6}$ & $1.42-20$ & $8.56 \mathrm{e}-21$ \\
\hline
\end{tabular}

The angular part can be expressed analytically in terms of Wigner-3j symbols [32], leading to

$$
\begin{aligned}
D_{a \rightarrow \epsilon, l, m}^{M} & =\sum_{l^{\prime}, m^{\prime}}(-1)^{m} \sqrt{(2 l+1)\left(2 l^{\prime}+1\right)} \\
& \times\left(\begin{array}{lll}
l^{\prime} & l & 1 \\
0 & 0 & 0
\end{array}\right)\left(\begin{array}{ccc}
l^{\prime} & l & 1 \\
-m^{\prime} & m & M
\end{array}\right) \\
& \times \int_{0}^{\infty} d r P_{a, l^{\prime}, m^{\prime}}(r) r P_{\epsilon, l}^{*}(r) .
\end{aligned}
$$

For the transitions considered in our computations, only the energy of the final continuum orbital is of relevance. Hence, we sum over $l$ and $m$ in equation (6) to obtain

$$
\sigma_{a \rightarrow \epsilon}=\frac{4}{3} \alpha \pi^{2} \omega N_{a} \sum_{l, m} \sum_{M=-1,0,1}\left|D_{a \rightarrow \epsilon, l, m}^{M}\right|^{2} .
$$

Finally, to obtain the single-photon ionisation cross section from a sub-shell $n l$, we sum $\sigma_{a \rightarrow \epsilon}$ over the orbitals $a$ in this sub-shell.

In Table 1, we compare our results for the single-photon ionisation cross sections of xenon with previous theoretical work [33]. We find a reasonable agreement, with the results in reference [33], which employs a HFS method for the computation of both bound and continuum orbitals. We employ a Hartree-Fock method to compute the bound states and a HFS method to compute the continuum orbitals. We find cross sections that are roughly $80 \%$ of the ones obtained in reference [33]. The agreement is not as good for the cross sections involving valence orbitals. The basis set we employ describes valence orbitals less accurately than inner-shell orbitals. However, for $850 \mathrm{eV}$ photon energy, single-photon ionisation cross sections of valence orbitals are very small compared to the ones of inner-shell orbitals rendering irrelevant the contribution of the former to our computations.

\subsection{Auger decay}

In general, the Auger rate is given by [34]

$$
\Gamma=\bar{\sum} 2 \pi|\mathcal{M}|^{2} \equiv \bar{\sum} 2 \pi\left|\left\langle\Psi_{\text {fin }}\left|H_{I}\right| \Psi_{\text {init }}\right\rangle\right|^{2},
$$

where $\bar{\sum}$ denotes a summation over the final states and an average over the initial states. $|\Psi\rangle$ is the wave function of all electrons in the atomic state. We assume that the Auger transition is a two-electron process, with $H_{I}$ being the electron-electron Coulomb repulsion term. We adapt to atoms the derivation used in our previous work for molecules [21]. We then find that the Auger rate involving two valence orbitals $a$ and $b$, an inner-shell orbital $c$, and a continuum orbital $\epsilon$ with quantum numbers $l, m$ is given by

$$
\begin{aligned}
& \mathcal{M}=\delta_{S^{\prime}, S} \delta_{M^{\prime}, M} \sum_{\substack{l_{c}, m_{c}, k \\
l_{a}, m_{a}, l_{b}, m_{b}}} \sum_{q=-k}^{k} \int d r_{1} \int d r_{2} \\
& (-1)^{m+m_{c}+q} \sqrt{(2 l+1)\left(2 l_{c}+1\right)\left(2 l_{b}+1\right)\left(2 l_{a}+1\right)} \\
& {\left[P_{\epsilon, l}\left(r_{1}\right) P_{c, l_{c}, m_{c}}\left(r_{2}\right) \frac{r_{<}^{k}}{r_{>}^{k+1}} P_{b, l_{b}, m_{b}}\left(r_{1}\right) P_{a, l_{a}, m_{a}}\left(r_{2}\right)\right.} \\
& \left(\begin{array}{lll}
l & k & l_{b} \\
0 & 0 & 0
\end{array}\right)\left(\begin{array}{ccc}
l & k & l_{b} \\
-m & -q & m_{b}
\end{array}\right)\left(\begin{array}{ccc}
l_{c} & k & l_{a} \\
0 & 0 & 0
\end{array}\right)\left(\begin{array}{ccc}
l_{c} & k & l_{a} \\
-m_{c} & q & m_{a}
\end{array}\right) \\
& +(-1)^{S} P_{\epsilon, l}\left(r_{1}\right) P_{c, l_{c}, m_{c}}\left(r_{2}\right) \frac{r_{<}^{k}}{r_{>}^{k+1}} P_{a, l_{a}, m_{a}}\left(r_{1}\right) P_{b, l_{b}, m_{b}}\left(r_{2}\right) \\
& \left.\left(\begin{array}{ccc}
l & k & l_{a} \\
0 & 0 & 0
\end{array}\right)\left(\begin{array}{ccc}
l & k & l_{a} \\
-m & -q & m_{a}
\end{array}\right)\left(\begin{array}{ccc}
l_{c} & k & l_{b} \\
0 & 0 & 0
\end{array}\right)\left(\begin{array}{ccc}
l_{c} & k & l_{b} \\
-m_{c} & q & m_{b}
\end{array}\right)\right],
\end{aligned}
$$

where $r_{<}=\min \left(r_{1}, r_{2}\right), r_{>}=\max \left(r_{1}, r_{2}\right)$. Moreover, $k$ and $q$ are the angular and magnetic quantum numbers, respectively, of the spherical harmonics involved in the multipole expansion of the Coulomb interaction, i.e. in the $1 / r_{12}$ term. The total Auger rate is given by

$$
\Gamma_{b, a \rightarrow c, \epsilon}=\sum_{\substack{S, M_{S} \\ S^{\prime}, M_{S}^{\prime}}} \pi N_{a b} N_{h} \sum_{l, m}|\mathcal{M}|^{2},
$$

where $N_{h}$ is the number of core holes in orbital c and $N_{a b}$ is the normalisation factor given by

$$
\begin{array}{rlr}
N_{a b} & =\frac{N_{a} N_{b}}{2 \times 2} & \text { valence electrons in different orbitals } \\
& =\frac{N_{a}\left(N_{a}-1\right)}{2 \times 2 \times 1} \quad \text { valence electrons in the same orbital. }
\end{array}
$$

$N_{a}$ and $N_{b}$ denote the occupation numbers of orbitals $a$ and $b$, respectively. Here, $S$ denotes the total spin of the two valence electrons, while $S^{\prime}$ denotes the total spin of the core electron plus the electron in the continuum. $M_{S}$ and $M_{S}^{\prime}$ denote their projections. To obtain the total Auger rate, $\Gamma_{s, t \rightarrow u, \epsilon}$ between $s, t, u$ sub-shells, we add the Auger rates $\Gamma_{b, a \rightarrow c, \epsilon}$ over the $a$ and $b$ orbitals in the respective $s, t$ sub-shells. We do not add over the $c$ orbitals in sub-shell $u$, since we average over the initial states.

In Tables 2 and 3, we compare our results for the Auger rates to previous calculations $[17,35]$. The Auger rates in reference [35] are computed using semi-empirical methods and in reference [17] using a non-relativistic HFS method. In Table 2, we find a close agreement with the results obtained in reference [17]. This is expected, since the techniques employed in our work are similar to the techniques used in reference [17]. In Table 3, the agreement with the results in reference [35] is reasonable given that the techniques employed in reference [35] are semi-empirical and 
Table 2. Comparison of Auger rates, expressed in units of $10^{-2}$ a.u. The valence electron refers to an electron in any of the sub-shells val $=4 s, 4 p, 4 d, 5 s$ or $5 p$.

\begin{tabular}{clll}
\hline$\Gamma_{s, t \rightarrow u, \epsilon}$ & Ref. [35] & Ref. [17] & This Work \\
\hline$u=3 s$ & & & \\
$\quad s=\mathrm{val}, t=\mathrm{val}$ & 2.06 & 1.85 & 1.71 \\
$\quad s=3 p, t=\mathrm{val}$ & 27.8 & 47.6 & 45.6 \\
$\quad s=3 d, t=\mathrm{val}$ & 7.59 & 8.98 & 8.61 \\
$u=3 p$ hole & & & \\
$\quad s=\mathrm{val}, t=\mathrm{val}$ & 2.18 & 2.10 & 2.03 \\
$\quad s=3 d, t=\mathrm{val}$ & 17.0 & 20.6 & 19.2 \\
$u=3 d$ hole & & & \\
$\quad s=$ val, $t=\mathrm{val}$ & 2.49 & 2.26 & 2.31 \\
\hline
\end{tabular}

Table 3. Comparison of Auger rates, expressed in units of $10^{-4}$ a.u. Each of the values given below corresponds to a sum of Auger rates, $\Gamma_{s, t \rightarrow u, \epsilon}$, over the relevant $s$ and $t$ sub-shells in the respective $n$ shells.

\begin{tabular}{cll}
\hline$\sum_{s, t} \Gamma_{s, t \rightarrow u, \epsilon}$ & Ref. [35] & This Work \\
\hline$u=2 s$ & & \\
$n=3,3$ & 491 & 465 \\
$n=4,4$ & 10.7 & 8.40 \\
$n=5,5$ & 0.078 & 0.043 \\
$n=3,4$ & 143 & 123.3 \\
$n=3,5$ & 12.4 & 7.79 \\
$n=4,5$ & 1.78 & 1.15 \\
$u=2 p$ & & \\
$n=3,3$ & 671 & 716 \\
$n=4,4$ & 15.3 & 12.6 \\
$n=5,5$ & 0.079 & 0.043 \\
$n=3,4$ & 201 & 189 \\
$n=3,5$ & 14.6 & 8.49 \\
$n=4,5$ & 3.99 & 1.22 \\
\hline
\end{tabular}

thus less accurate than the techniques employed in the current study.

\subsection{Shake-off}

Following single-photon ionisation, if the photo-electron escapes with high energy, the sudden change in the potential energy can result in the ionisation of one more electron. According to the sudden approximation $[36,37]$ the probability for an electron to be shaken off from a $u$ subshell is given by

$$
P_{u}^{S} \approx 1-\left[\left|\int \phi_{a}^{*}(\mathbf{r}) \phi_{a}^{\prime}(\mathbf{r}) d \mathbf{r}\right|^{2}\right]^{N_{u}},
$$

where $\phi_{a}$ and $\phi_{a}^{\prime}$ are the orbital eigenstates of the Hamiltonian before and after single-photon ionisation takes place, with $a$ an orbital of the $u$ sub-shell and $N_{u}$ the occupancy of the sub-shell. Note that in equation (12), we neglect shake-up processes involving transitions of an electron to a bound orbital that differs from $a$.

\subsection{Monte-Carlo technique}

This method involves starting from the ground state of xenon and propagating forward in time with a time step, $\delta t=0.01 \mathrm{fs}$. We have checked that the Monte-Carlo technique converges for the chosen time step. At each time step, we consider all energetically allowed transitions from the current state $i$ to a state $j$. We then compute the rates associated with these allowed transitions, as follows

$$
\begin{aligned}
w_{i \rightarrow j}(t) & =\sigma_{i \rightarrow j} J(t) & & \text { photo ionisation } \\
& =\sigma_{i \rightarrow j} P^{S} J(t) & & \text { photo ionisation } \\
& =\Gamma_{i \rightarrow j} & & \text { Auger decay. }
\end{aligned}
$$

We do not include fluorescence in our calculations, since the fluorescence rates are found to be much lower than the Auger rates [17]. Assuming that each of these processes follows an exponential decay law [19], we assign a time to each allowed transition as follows

$$
t_{i \rightarrow j}(t)=-\ln (\chi) / w_{i \rightarrow j}(t),
$$

where $\chi$ is a random number between zero and one. If the smallest time $t_{i \rightarrow j}$ associated with these transitions is less than the propagation time step, we register the transition associated with the smallest time. Next, we repeat the process starting from time $t+t_{i \rightarrow j}$ with xenon in state $j$. Following this process, we propagate forward until reaching asymptotic times. The above described process corresponds to one realisation of the Monte-Carlo method. We implement this method $10^{5}$ times, which we find to be a sufficient number of realisations to achieve convergence.

\section{Results}

We first compare our results for the final ion yields of xenon driven by an $850 \mathrm{eV}$ FEL pulse with the experimental results obtained in reference [20]. To do so, we consider a peak intensity $I_{0}$ equal to $5.6 \times 10^{16} \mathrm{Wcm}^{-2}$ and a fullwidth half-maximum $\tau$ equal to $150 \mathrm{fs}$, as in reference [20]. Moreover, to obtain the ion yields and the relevant pathways, we average over the transverse, $(x, y)$ plane, profile of the beam, disregarding the beam's z-dependence. This is in accord with reference [38], where it is explicitly stated that the photon flux employed describes the beam profile used in the experimental work described in references $[20,39]$. Thus, to compare our results with the experimental ones in reference [20], we use the photon flux described in reference [38]. The latter is given by

$$
J(x, y, t)=I_{0} \exp \left(-4 \ln 2\left[\left(x / \rho_{x}\right)^{2}+\left(y / \rho_{y}\right)^{2}+(t / \tau)^{2}\right]\right) / \omega,
$$

with $\rho_{x}=2.2 \mu \mathrm{m}$ and $\rho_{y}=1.2 \mu \mathrm{m}$. We compute the ion yields for the intensities corresponding to $\left(10^{3}\right)^{2}$ points on a square grid with dimensions $(10 \mu \mathrm{m})^{2}$ and average over the grid area.

Our results for the final ion yields are shown in Figure 1. The yields of the ion states $\mathrm{Xe}^{q+}$ with $q=1,2,3$ are very small. In contrast, the yields of the ion states with 


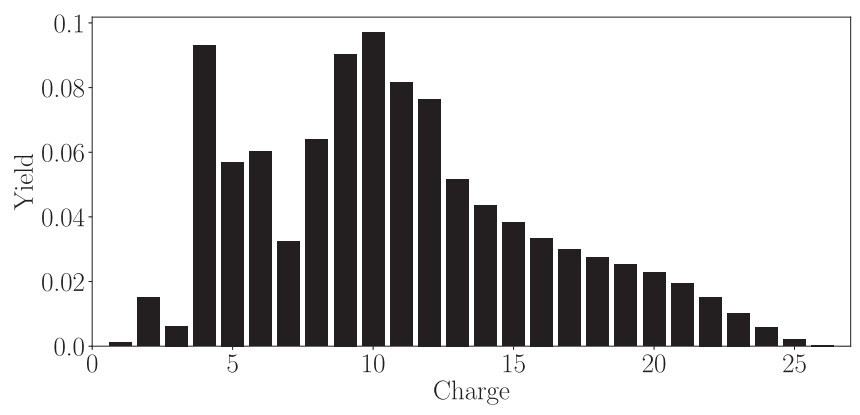

Fig. 1. Final ion yields of xenon interacting with an $850 \mathrm{eV}$ FEL pulse of peak intensity $5.6 \times 10^{16} \mathrm{Wcm}^{-2}$ and duration $150 \mathrm{fs}$. The spatial distribution of the laser pulse is taken into account.

$q=4,5,6$ as well as with $q=8-13$ are significant. We also find that the highest-charged state reaches up to $q=26$, in accord with the single-photon ionisation processes occurring up to charge $q=25$. These results are in agreement with the experimental results in reference [20]. To identify the reason for the high yields of the ion states with $q$ around 10, we compare these ion yields and pathways in Figure 2a with those in Figure $2 \mathrm{~b}$ when xenon is driven by an FEL pulse of smaller peak intensity of $10^{15} \mathrm{Wcm}^{-2}$. In Figures 2a and $2 \mathrm{~b}$, in addition to the ion yields, we show, for each ion state, the contribution of pathways that differ in the number of single-photon absorptions that occur. We find that the larger ion yields of the states with q equal or greater than 10 are due to the higher number of photons absorbed for the higher intensity pulse. For instance, the ion state with $q=10$ is mostly populated by pathways involving four single-photon ionisation processes, for the higher intensity pulse, versus three, for the lower intensity one. Moreover, the ion state with $q=12$ has a significant yield only for the higher intensity pulse, due to the large number, equal to five, of single-photon ionisations taking place. Hence, the pulse of the higher peak intensity has higher ion yields for the highly-charged ion states due to the larger number of single-photon absorptions that occur. This result comes as no surprise and has been reported in several previous studies $[13-16,40]$.

We also find that larger yields for highly-charged ion states can be achieved by a lower intensity and longer duration $850 \mathrm{eV}$ FEL pulse when comparing with a higher intensity and smaller duration $850 \mathrm{eV}$ FEL pulse. Both pulses have the same pulse energy. Surprisingly, we find that this is not due to a higher number of single-photon absorptions taking place. Quite the contrary. Indeed, in Figures $3 \mathrm{a}-3 \mathrm{c}$ we show the ion yields for decreasing pulse intensity and increasing pulse duration. Moreover, as in Figure 2, we show, for each ion state, the contribution of pathways that differ in the number of single-photon absorptions that occur. Comparing the ion yields of the highly-charged states, we find that as the pulse duration increases a given ion state with charge 7 and higher is reached via less single-photon absorptions while the ion yield of this state increases. For instance, for the ion state with $q=7$ the prevalent pathway for the 5 fs pulse duration involves 3 single-photon absorptions while for the $250 \mathrm{fs}$ pulse duration it involves 2 single-photon absorp-
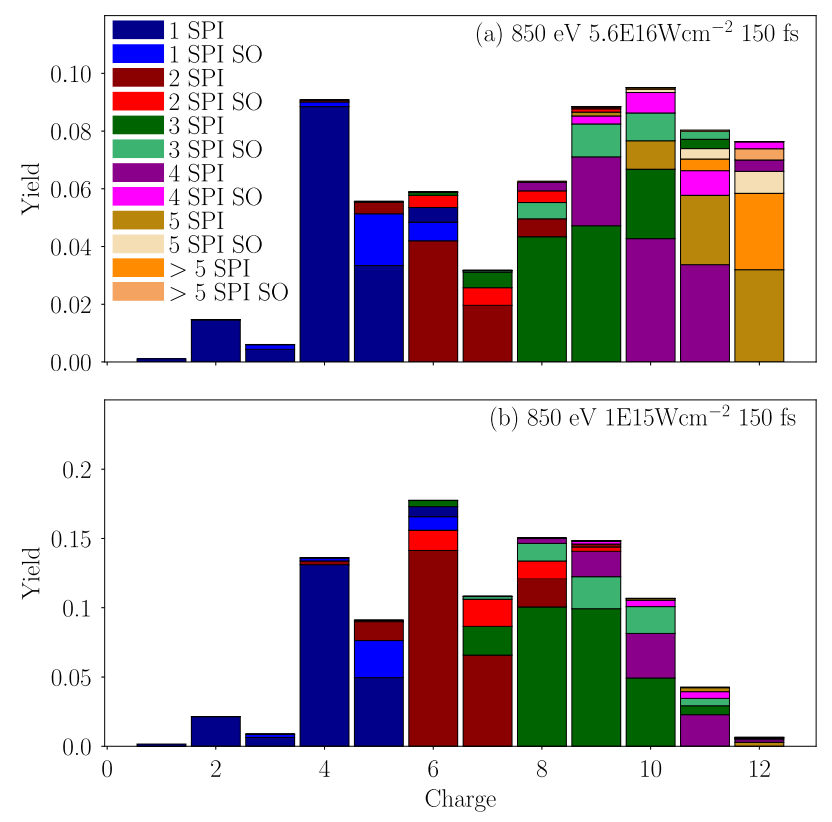

Fig. 2. Final ion yields of xenon interacting with an $850 \mathrm{eV}$ FEL pulse of duration $150 \mathrm{fs}$. For each ion state, we show the contribution of pathways separated according to the number of single-photon ionisation transitions that take place. (a) An FEL pulse of peak intensity $5.6 \times 10^{16} \mathrm{Wcm}^{-2}$ is considered and the results account for the spatial distribution of the pulse; (b) an FEL pulse of peak intensity $10^{15} \mathrm{Wcm}^{-2}$ is considered without accounting for the spatial distribution of the pulse. SPI stands for single-photon ionization, while SO stands for shake-off.

tions. However, the ion yield of the $q=7$ state is larger for the longer pulse. Similarly, for the ion state with $q=10$ the prevalent pathway for the 5 fs pulse duration involves 4 single-photon absorptions while for the $250 \mathrm{fs}$ pulse duration it involves 3 single-photon absorptions. However, the ion yield of the $q=10$ state is larger for the longer pulse. This finding is consistent with our finding that the population of ion states that is formed by absorbing a given number of photons is roughly the same for all three pulses. In addition, the ion states with charge $q$ up to 5 are formed mainly by a single-photon absorption. It then follows, that since for the longer duration pulse we have larger yields for the highly-charged states, the latter are formed by absorption of more photons for the shorter duration pulse.

We find that (not shown) more Auger processes take place between two subsequent single-photon absorption processes when xenon interacts with the long duration pulses. This is the reason the yields of the highly-charged ion states for the $50 \mathrm{fs}$ and $250 \mathrm{fs}$ FEL pulses are larger compared to the $5 \mathrm{fs}$ pulse. We explain this in what follows. Once a $3 d$ electron ionizes, following a single-photon absorption, then, for all three duration pulses we consider, an Auger process takes place with an electron from the $n=4$ shell dropping in to fill the vacancy in the $3 d$ shell. However, the rate for a $3 d$ electron to absorb a photon is much smaller, by an order of magnitude, for the $50 \mathrm{fs}$ pulse compared to the $5 \mathrm{fs}$ one; this rough estimate assumes absorption takes place at the peak of the pulses. 

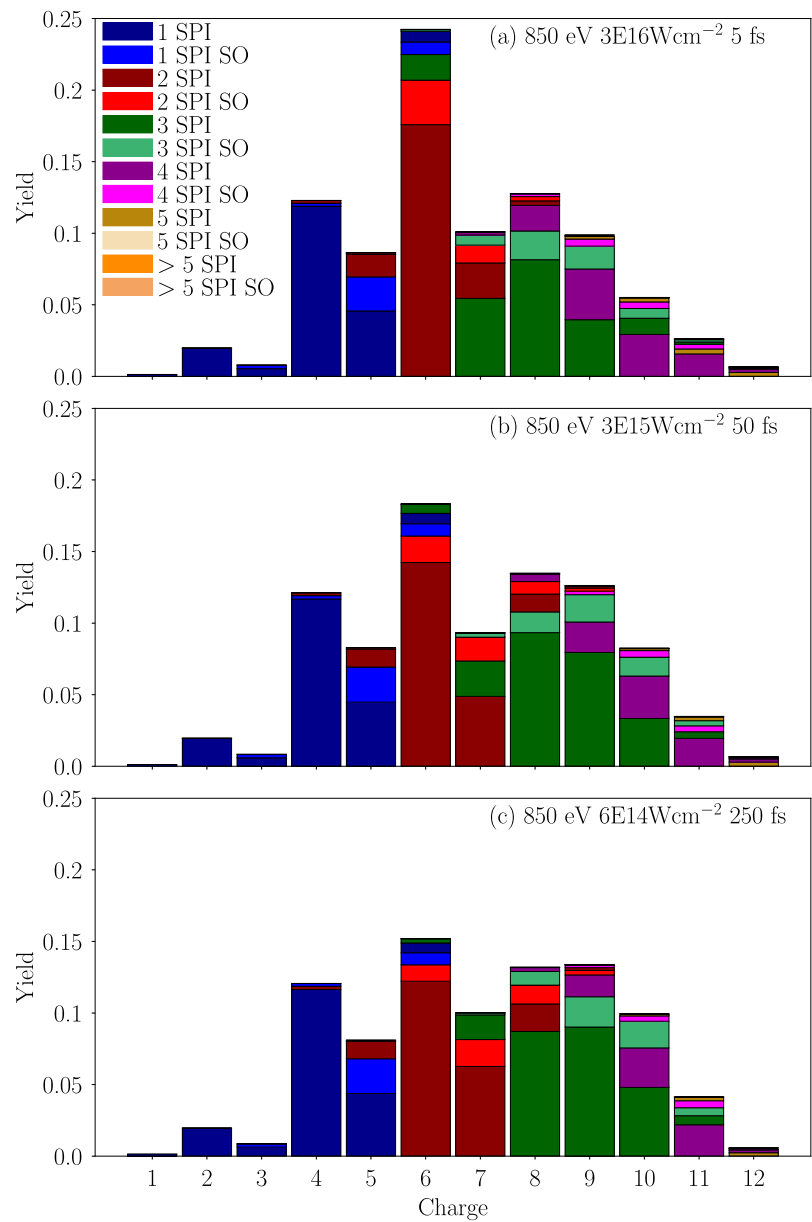

Fig. 3. Final ion yields of xenon interacting with $850 \mathrm{eV} \mathrm{FEL}$ pulses of fixed pulse energy with varying duration. For each ion state, we show the contribution of pathways separated according to the number of single-photon ionisation transitions that take place. SPI stands for single-photon ionization, while SO stands for shake-off.

For the shorter pulse, this absorption rate is larger than the Auger rate where an electron in the $n=5$ shell drops in to fill the vacancy in the $n=4$ shell. It is the other way around for the longer duration pulse. It then follows that Auger cascades are more likely to occur between subsequent single-photon absorptions for the longer duration pulses. We demonstrate this in Figure 4, where we plot for each final charged ion state the contribution of states with a certain sub-shell vacancy. For instance, for charged states up to $q=5$, we find that each ion state has electrons missing mostly from the $5 p$ sub-shell, and less so from the $5 s$ sub-shell. For each final ion state with charge $q$ equal to 6 and higher, we find that the probability for a $4 d$ subshell vacancy is higher for the shorter duration pulse. This finding is consistent with our finding that Auger cascades are less likely for the shorter duration pulse, thus resulting in a higher chance for an $n=4$ vacancy in the final ion state. We note that for all three pulse durations considered the vast majority of pathways leading to the formation of each ion state involves only a single core hole at any given time. As a result, the smaller yields we find for the highly-

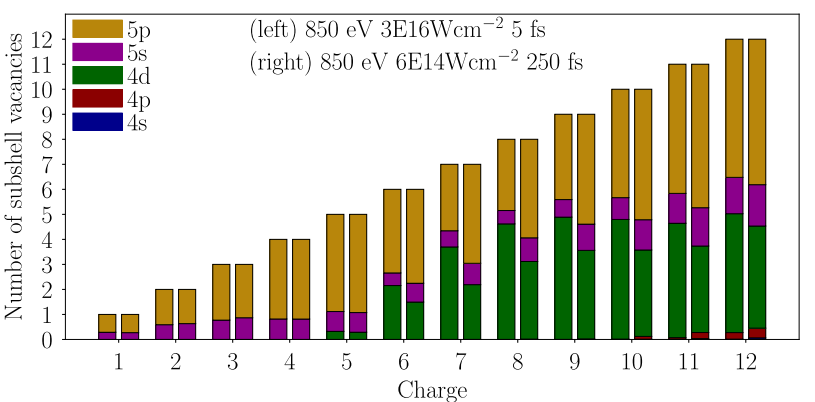

Fig. 4. Contribution of states with a certain sub-shell vacancy for each final ion state of xenon interacting with two FEL pulses.

charged states for the short duration pulse are not related to an atom becoming temporarily transparent [41].

\section{Conclusions}

In this work, we compute single-photon ionisation crosssections and Auger rates for xenon, by adapting to atoms the formalism we have previously developed for diatomic molecules [21]. In the non-relativistic regime, this formalism is general and is applicable to complex multi-electron atoms where the orbital wave functions are accurately described by many $l$ quantum numbers. Thus, our formalism is more accurate than previous models which describe the orbital wave functions with only one $l$ quantum number. Our results for the ion yields produced when xenon interacts with an $850 \mathrm{eV}$ FEL pulse are in good agreement with previous experimental results. Moreover, we have identified the prevalent pathways for the ion states of xenon driven by three FEL pulses of the same pulse energy and different pulse duration. We find that the longer duration pulse has larger yields for the highly-charged ion states compared to the shorter duration one. We find that the larger yield for a given highly-charged state is not due to more single-photon absorptions for the longer duration pulse. We find instead that it is due to a larger number of Auger decays occurring between subsequent singlephoton ionisation events in the case of the longer duration pulse.

\section{Author contribution statement}

All the authors were involved in the preparation of the manuscript. All the authors have read and approved the final manuscript.

Publisher's Note The EPJ Publishers remain neutral with regard to jurisdictional claims in published maps and institutional affiliations.

Open Access This is an open access article distributed under the terms of the Creative Commons Attribution License (https://creativecommons.org/licenses/by/4.0/), which permits unrestricted use, distribution, and reproduction in any medium, provided the original work is properly cited. 


\section{References}

1. C. Pellegrini, Eur. Phys. J. H 37, 659 (2012)

2. C. Bostedt, J. Bozek, P.H. Bucksbaum, R. Coffee, J.B. Hastings, Z. Huang, R. Lee, S. Schorb, J.N. Corlett, P. Denes, P. Emma, R. Falcone, R.W. Schoenlein, G. Doumy, E. Kanter, B. Kraessig, S. Southworth, L. Young, L. Fang, M. Hoener, N. Berrah, C. Roedig, L. DiMauro, J. Phys. B: At., Mol. Opt. Phys. 46, 164003 (2013)

3. P. Emma, R. Akre, J. Arthur, R. Bionta, C. Bostedt, J. Bozek, A. Brachmann, P. Bucksbaum, R. Coffee, F. Decker, Y. Ding, D. Dowell, S. Edstrom, A. Fisher, J. Frisch, S. Gilevich, J. Hastings, G. Hays, P. Hering, Z. Huang, R. Iverson, H. Loos, M. Messerschmidt, A. Miahnahri, S. Moeller, H. Nuhn, G. Pile, D. Ratner, J. Rzepiela, D. Schultz, T. Smith, P. Stefan, H. Tompkins, J. Turner, J. Welch, W. White, J. Wu, G. Yocky, J. Galayda, Nat. Photonics 4(9), 641 (2010)

4. J.P. Marangos, Contemp. Phys. 52(6), 551 (2011)

5. J. Ullrich, A. Rudenko, R. Moshammer, Annu. Rev. Phys. Chem. 63, 635 (2012)

6. I. Schlichting, J. Miao, Curr. Opin. Struct. Biol. 22, 613 (2012)

7. R. Neutze, R. Wouts, D. van der Spoel, E. Weckert, J. Hajdu, Nature 406, 752 (2000)

8. L. Redecke, K. Nass, D.P. DePonte, T.A. White, D. Rehders, A. Barty, F. Stellato, M. Liang, T.R. Barends, S. Boutet, G.J. Williams, M. Messerschmidt, M.M. Seibert, A. Aquila, D. Arnlund, S. Bajt, T. Barth, M.J. Bogan, C. Caleman, T.C. Chao, R.B. Doak, H. Fleckenstein, M. Frank, R. Fromme, L. Galli, I. Grotjohann, M.S. Hunter, L.C. Johansson, S. Kassemeyer, G. Katona, R.A. Kirian, R. Koopmann, C. Kupitz, L. Lomb, A.V. Martin, S. Mogk, R. Neutze, R.L. Shoeman, J. Steinbrener, N. Timneanu, D. Wang, U. Weierstall, N.A. Zatsepin, J.C.H. Spence, P. Fromme, I. Schlichting, M. Duszenko, C. Betzel, H.N. Chapman, Science 339, 227 (2013)

9. R. Santra, N.V. Kryzhevoi, L.S. Cederbaum, Phys. Rev. Lett. 103, 013002 (2009)

10. L.S. Cederbaum, F. Tarantelli, A. Sgamellotti, J. Schirmer, J. Chem. Phys. 85(11), 6513 (1986)

11. L.S. Cederbaum, F. Tarantelli, A. Sgamellotti, J. Schirmer, J. Chem. Phys. 86(4), 2168 (1987)

12. A.A. Sorokin, S.V. Bobashev, T. Feigl, K. Tiedtke, H. Wabnitz, M. Richter, Phys. Rev. Lett. 99, 213002 (2007)

13. B. Rudek, S.K. Son, L. Foucar, S.W. Epp, B. Erk, R. Hartmann, M. Adolph, R. Andritschke, A. Aquila, N. Berrah, C. Bostedt, J. Bozek, N. Coppola, F. Filsinger, H. Gorke, T. Gorkhover, H. Graafsma, L. Gumprecht, A. Hartmann, G. Hauser, S. Herrmann, H. Hirsemann, P. Holl, A. Hömke, L. Journel, C. Kaiser, N. Kimmel, F. Krasniqi, K.U. Kühnel, M. Matysek, M. Messerschmidt, D. Miesner, T. Möller, R. Moshammer, K. Nagaya, B. Nilsson, G. Potdevin, D. Pietschner, C. Reich, D. Rupp, G. Schaller, I. Schlichting, C. Schmidt, F. Schopper, S. Schorb, C.D. Schröter, J. Schulz, M. Simon, H. Soltau, L. Strüder, K. Ueda, G. Weidenspointner, R. Santra, J. Ullrich, A. Rudenko, D. Rolles, Nat. Photonics 6, 858 (2012)

14. M.G. Makris, P. Lambropoulos, A. Mihelič, Phys. Rev. Lett. 102, 033002 (2009)

15. P. Lambropoulos, K. Papamihail, P. Decleva, J. Phys. B: At., Mol. Opt. Phys. 44, 175402 (2011)
16. K. Toyota, S.K. Son, R. Santra, Phys. Rev. A 95 (2017)

17. S.K. Son, R. Santra, Phys. Rev. A 85, 063415 (2012)

18. L. Madsen, P. Lambropoulos, Phys. Rev. A 59 (1999)

19. Z. Jurek, S.K. Son, B. Ziaja, R. Santra, J. Appl. Crystallogr. 49, 1048 (2016)

20. H. Thomas, A. Helal, K. Hoffmann, N. Kandadai, J. Keto, J. Andreasson, B. Iwan, M. Seibert, N. Timneanu, J. Hajdu, M. Adolph, T. Gorkhover, D. Rupp, S. Schorb, T. Möller, G. Doumy, L.F. DiMauro, M. Hoener, B. Murphy, N. Berrah, M. Messerschmidt, J. Bozek, C. Bostedt, T. Ditmire, Phys. Rev. Lett. 108, 133401 (2012)

21. H.I.B. Banks, D.A. Little, J. Tennyson, A. Emmanouilidou, Phys. Chem. Chem. Phys. 19, 19794 (2017)

22. A.O.G. Wallis, L. Lodi, A. Emmanouilidou, Phys. Rev. A 89, 063417 (2014)

23. A.O.G. Wallis, H.I.B. Banks, A. Emmanouilidou, Phys. Rev. A 91, 063402 (2015)

24. S.K. Son, L. Young, R. Santra, Phys. Rev. A 83, 033402 (2011)

25. C.P. Robert, G. Casella, Monte Carlo Statistical Methods (Springer Texts in Statistics) (Springer-Verlag, Berlin, Heidelberg, 2005)

26. J.J. Sakurai, Modern Quantum Mechanics (AddisonWesley, 1994)

27. H.J. Werner, P.J. Knowles, R. Lindh, F.R. Manby, M. Schütz, et al. MOLPRO, a package of ab initio programs (2010), http://www.molpro.net/

28. L. Martins, F. de Souza, G. Ceolin, F. Jorge, R. de Berrêdo, C. Campos, Comput. Theor. Chem. 1013, 62 (2013)

29. B.V. Noumerov, Mon. Not. R. Astron. Soc. 84, 592 (1924)

30. F. Herman, S. Skillman, Atomic structure calculations (Prentice-Hall, New Jersey, 1963)

31. M.D. Pauli, Herman-Skillman program (2001), Hermes . phys.uwm.edu/projects/elecstruct/elecstruct.html

32. A.R. Edmonds, Angular Momentum in Quantum Mechanics (Princeton University Press, 1960)

33. J. Yeh, I. Lindau, At. Data Nucl. Data Tables 32, 1 (1985)

34. W. Pauli, Wave Mechanics: Volume 5 of Pauli Lectures on Physics (Wiley, 2000)

35. E.J. McGuire, Phys. Rev. A 5, 1043 (1972)

36. T. Åberg, Ann. Acad. Sci. Fenn. Ser. A VI 308, 1 (1969)

37. T.A. Carlson, C.W. Nestor, Phys. Rev. A 8, 2887 (1973)

38. C. Buth, J.C. Liu, M.H. Chen, J.P. Cryan, L. Fang, J.M. Glownia, M. Hoener, R.N. Coffee, N. Berrah, J. Chem. Phys. 136, 214310 (2012)

39. M. Hoener, L. Fang, O. Kornilov, O. Gessner, S.T. Pratt, M. Gühr, E.P. Kanter, C. Blaga, C. Bostedt, J.D. Bozek, P.H. Bucksbaum, C. Buth, M. Chen, R. Coffee, J. Cryan, L. DiMauro, M. Glownia, E. Hosler, E. Kukk, S.R. Leone, B. McFarland, M. Messerschmidt, B. Murphy, V. Petrovic, D. Rolles, N. Berrah, Phys. Rev. Lett. 104, $253002(2010)$

40. K. Motomura, H. Fukuzawa, S.K. Son, S. Mondal, T. Tachibana, Y. Ito, M. Kimura, K. Nagaya, T. Sakai, K. Matsunami, S. Wada, H. Hayashita, J. Kajikawa, X.J. Liu, R. Feifel, P. Johnsson, M. Siano, E. Kukk, B. Rudek, B. Erk, L. Foucar, E. Robert, C. Miron, K. Tono, Y. Inubushi, T. Hatsui, M. Yabashi, M. Yao, R. Santra, K. Ueda, J. Phys. B: At. Mol. Opt. Phys. 46, 164024 (2013)

41. U. Saalmann, J.M. Rost, Phys. Rev. Lett. 89, 143401 (2002) 